\title{
Geometrical framework for picture changing operators in the pure spinor formalism
}

\section{Andrei Mikhailov and Dennis Zavaleta}

Instituto de Fisica Teorica, Universidade Estadual Paulista, R. Dr. Bento Teobaldo Ferraz 271, Bloco II - Barra Funda CEP:01140-070 - Sao Paulo, Brasil E-mail: a.mikhaylov@unesp.br, dennis.zavaleta@unesp.br

ABSTRACT: It is well known in NSR string theory, that vertex operators can be constructed in various "pictures". Recently this was discussed in the context of pure spinor formalism. NSR picture changing operators have an elegant super-geometrical interpretation. In this paper we provide a generalization of this super-geometrical construction, which is also applicable to the pure spinor formalism.

KEYwords: Superstrings and Heterotic Strings, AdS-CFT Correspondence

ARXIV EPRINT: 2003.13995 


\section{Contents}

1 Introduction 1

2 Geometrical interpretation of picture changing 3

2.1 Reminder on odd tangent bundle ПТX 3

2.2 Picture raising operators 3

2.3 Some properties of $\Pi T \quad 4$

3 Submanifolds in $\Pi T X$

4 Pure spinor target space as a subspace in $\Pi T X \quad 5$

4.1 Supersymmetry generators and invariant derivatives 5

4.2 Description of $C \subset \Pi T X \quad 6$

$\begin{array}{lll}4.3 & \text { Solving the pure spinor constraint } & 7\end{array}$

5 An action of $C \mathbf{R}^{0 \mid 8}$ on $C \quad 8$

5.1 Type IIB supergravity vertex operator at picture $(-8) \quad 9$

$\begin{array}{lll}5.2 & \text { Type IIB supergravity vertex operator at picture (0) } & 10\end{array}$

6 Open questions 12

A Anticommuting $\nabla_{-}$and $\nabla_{+} \quad 12$

\section{Introduction}

Pure spinor formalism is very promising for studying strings in AdS, since it naturally includes the Ramond-Ramond fields [1,2]. However, the progress has been slowed down by the lack of explicit formula for vertex operators (see [3, 4] for definitions and [5] for a simple example). Recently, a promising new method was suggested in [6]. The main idea is to construct the vertex in nonzero picture. ${ }^{1}$ This means allowing delta-functions of the pure spinor ghosts. It was shown in [6], that the ansatz for $1 / 2$-BPS vertex is simplified in the -8 picture. Then, the vertex operator in picture zero can be obtained by applying picture raising operators, as explained in [10]. However, this picture-raising procedure is not the usual one [11], because the pure spinor variables are constrained to live on a cone. In particular, it does not immediately fit into the geometrical framework of $[12,13]$.

In this paper we will develop a generalization of the approach of $[12,13]$, which does cover the construction of $[6,10]$.

\footnotetext{
${ }^{1}$ Picture changing operators were previously discussed in the context of pure spinor formalism in $[7,8]$. More generally, the target space picture changing operators were essential in constructing the Chern-Simons actions in [9].
} 
The pure spinor target space can be considered a generalization of the odd tangent bundle $\Pi T X$ over the super-space-time $X$ (see section 2.1). The generalization consists of imposing some constraints on the coordinates in the fiber. If $X$ has coordinates $x, \theta$, then $\Pi T X$ has coordinates $x, \theta, d x, d \theta$. We consider a submanifold $C \subset \Pi T X$ defined by some quadratic and linear equations - see section 4 and [14]. The idea is to construct the action of some "odd loop group" ПTG on $C$ and then average over its orbits, using the natural measure on ПТG. The integration removes the delta-functions of the pure spinor ghosts, and the result is the picture zero vertex operator.

One lesson from our study: it is useful to consider pure spinor vertex operators as, roughly speaking, differential forms on space-time:

- vertex operators are (pseudo) differential forms

The pure spinor variable $\lambda$ is, in some sense, $d \theta$ - the differential of the fermionic coordinate of the target space, with the pure spinor constraint imposed [14]. The de Rham operator $d$ is identified with the BRST operator $Q$. In this language picture changing operators are geometrical operations on forms, basically averaging over an orbit of some group. We want this operation to commute with the action of $Q=d$. To achieve that, we take the group to be of the form $\Pi T G$ for some Lie supergroup $G$, and construct its action in such a way that $d$ agrees with the nilpotent vector field of $\Pi T G$, as explained in section 2.2. The operation of averaging is usually non-local, an integral transform. But it becomes local if all integrations are absorbed by delta-functions. We do not want to allow delta-functions of the target space coordinates, because we consider vertex operators corresponding to smooth supergravity solutions. Instead, we introduce delta-functions of $d \theta$, which means allowing "pseudo-differential forms". It seems that our construction requires $G$ to be purely odd supergroup (in particular, abelian). Basically, it is $\mathbf{R}^{0 \mid 8}$. If bosonic directions were present in $G$, then integration over them would not be absorbed by delta-functions, leading to non-locality.

The main point of our approach is defining a structure of differential $\mathbf{R}^{0 \mid 8}$-module on the space of vertex operators, section 3. The construction is nontrivial, and "depends on some luck", section 5. We hope that it would generalize to the case of pure spinor superstring in AdS, but at this time we do not have such generalization.

It is also worth noting that this formalism has also been applied to the superstring in other dimensions (e.g. $D=3$ in [7]) where there are no pure spinor constraints and so the delta functions on these variables does not present any problem.

Plan of this paper. We first review the geometrical construction of $[12,13]$ in section 2, and discuss $d$-closed submanifolds in section 3 . Then we apply these concepts to pure spinor formalism in section 4. In section 5 and section $\mathrm{A}$ we introduce a generalization of the geometrical picture changing procedure of $[12,13]$ and reproduce the result of $[10]$. Finally, in section 6 we discuss open questions. 


\section{Geometrical interpretation of picture changing}

\subsection{Reminder on odd tangent bundle $\Pi T X$}

When studying a supermanifold $X$, it is often useful to consider, for any "test" supermanifold $S$, the space of maps:

$$
\mathcal{F}_{X}[S]=\operatorname{Map}(S, X)
$$

(see J. Bernstein's lectures in [16], which are also available online at https://www.math.ias.edu/QFT/fall/). This defines a contravariant functor $S \mapsto \mathcal{F}_{X}[S]$ from supermanifolds to sets. This is the "functor of points"; $\mathcal{F}_{X}[S]$ is called $S$-points of $X$.

If $X$ is a supermanifold, then functor $\Pi T$ in the category of supermanifolds is defined as follows:

$$
\operatorname{Map}(S, \Pi T X)=\operatorname{Map}\left(S \times \mathbf{R}^{0 \mid 1}, X\right)
$$

Functions on $\Pi T X$ are called "pseudo-differential forms (PDFs) on $X$ ".

In particular, for a Lie supergroup $G$, the odd tangent space $\Pi T G$ is also a Lie supergroup, which might be considered an odd analogue of the loop group of $G$. Let $\mathbf{g}=\operatorname{Lie}(G)$ be the Lie algebra of $G$. The Lie algebra of $\Pi T G$ is usually called "cone of $\mathbf{g}$ " and denoted $C \mathbf{g}$ :

$$
C \mathbf{g}=\operatorname{Lie\Pi TG}
$$

\section{$2.2 \quad$ Picture raising operators}

Suppose that a Lie supergroup $G$ acts on a supermanifold $X$. Then $\Pi T G$ acts on $\Pi T X$. This means that pseudodifferential forms on $X$ form a linear representation of $\Pi T G$. Given a PDF $\omega \in \operatorname{Fun}(\Pi T X)$ we consider:

$$
\Gamma \omega=\int_{g \in \Pi T G} \omega \circ g
$$

We restrict ourselves to those $\omega$ for which this integral converges. We observe that:

$$
\begin{aligned}
(\Gamma \omega) \circ g & =0 \quad \forall \quad g \in \Pi T G \\
d \Gamma \omega & =\Gamma d \omega
\end{aligned}
$$

Eq. (2.5) implies that $\omega$ actually descends (as a PDF) on the space of orbits of $G$ in $X$. Eq. (2.6) implies that closed $\omega$ gives closed $\Gamma \omega$.

This construction is usually applied to the case when $G$ is purely odd, i.e. take the simplest example $G=\mathbf{R}^{0 \mid 1}$ and $\Pi T G=\mathbf{R}^{1 \mid 1}$ with the target supermanifold $X$ parametrized by $\left(Z^{M}\right)$. Suppose that $\omega$ contains enough delta-functions to absorb the integration along odd variables. In this case the convergence of the integral in eq. (2.4) is guaranteed, and moreover $\omega \mapsto \Gamma \omega$ is actually a local operation. To be explicit, suppose that the Lie algebra $\mathbf{g}=\mathbf{R}^{0 \mid 1}$ is generated by the odd vector fields $\nu$ :

$$
\nu=\nu^{M}(Z) \frac{\partial}{\partial Z^{M}}
$$


The action of the group $\mathbf{R}^{0 \mid 1} \times X \rightarrow X$ is then given by:

$$
\left(\epsilon, Z^{M}\right) \mapsto Z^{M}+\epsilon \nu^{M}
$$

The corresponding action of $\Pi T G$ on $\Pi T X$ is:

$$
\begin{aligned}
a: \Pi T G \times \Pi T X & \rightarrow \Pi T X \\
\left(\epsilon, d \epsilon, Z^{M}, d Z^{M}\right) & \stackrel{a}{\mapsto}\left(Z^{M}+\epsilon \nu^{M}, d Z^{M}+d \epsilon \nu^{M}-\epsilon d \nu^{M}\right)
\end{aligned}
$$

The integral of eq. (2.4) is:

$$
\left(\Gamma_{\nu} \omega\right)(Z, d Z)=\int D(d \epsilon) D(\epsilon) \omega\left(Z^{M}+\epsilon \nu^{M}, d Z^{M}+d \epsilon \nu^{M}-\epsilon d \nu^{M}\right)
$$

For any supermanifold $M$, the odd tangent space $\Pi T M$ has a canonical vector field $d$. If we thing of $\Pi T M$ as the space of maps $\mathbf{R}^{0 \mid 1} \rightarrow M$, then this $d$ is the infinitesimal shift in $\mathbf{R}^{0 \mid 1}$. Eq. (2.6) can be derived by observing that for any $\omega$, a function on $\Pi T X$ :

$$
\left(d_{\Pi T G}+d_{\Pi T X}\right)(\omega \circ a)=\left(d_{\Pi T X} \omega\right) \circ a
$$

After integrating on the odd parameter $\epsilon$, we can arrive at the "usual" (in string theory literature) expression for the PCO:

$$
\left(\Gamma_{\nu} \omega\right)=\left[d, \Theta\left(\iota_{\nu}\right)\right]
$$

where $\iota_{\nu}=\nu^{M} \frac{\partial}{\partial d Z^{M}}$.

This can be generalized to $G=\mathbf{R}^{0 \mid n}$ and $\Pi T G=\mathbf{R}^{n \mid n}$ where $\mathbf{g}=\mathbf{R}^{0 \mid n}$ is generated by $n$ odd vector fields $\nu_{a}$ with $a=1, \ldots, n$. Then the integral becomes

$$
\left(\Gamma_{\nu} \omega\right)(Z, d Z)=\int \prod_{a} D\left(d \epsilon^{a}\right) \prod_{a} D\left(\epsilon^{a}\right) \omega\left(g^{\epsilon^{a}, d \epsilon^{a}}(Z, d Z)\right)
$$

where $g^{\epsilon^{a}, d \epsilon^{a}}$ is the flow corresponding to the infinitesimal transformation

$$
\left(\epsilon^{a}, d \epsilon^{a}, Z^{M}, d Z^{M}\right) \mapsto\left(Z^{M}+\epsilon^{a} \nu_{a}^{M}, d Z^{M}+d \epsilon^{a} \nu_{a}^{M}-\epsilon^{a} d \nu_{a}^{M}\right)
$$

\subsection{Some properties of $\Pi T$}

Some computations are simplified (see section 5) by considering the iterated application of $\Pi T$. Consider

$$
\mu: \Pi T \Pi T X \rightarrow \Pi T X
$$

induced by the diagonal map

$$
\Delta: \mathbf{R}^{0 \mid 1} \longrightarrow \mathbf{R}^{0 \mid 1} \times \mathbf{R}^{0 \mid 1}
$$

inducing

$$
\begin{aligned}
\mu[S]: \operatorname{Map}\left(S \times \mathbf{R}^{0 \mid 1} \times \mathbf{R}^{0 \mid 1}, X\right) & \longrightarrow \operatorname{Map}\left(S \times \mathbf{R}^{0 \mid 1}, X\right) \\
\phi & \longmapsto \mu[S](\phi)=\phi \circ(\mathrm{id} \times \Delta)
\end{aligned}
$$

There is a canonical nilpotent odd vector field $d \in \operatorname{Vec}(\Pi T X)$ generating shifts along $\mathbf{R}^{0 \mid 1}$. 
For any odd vector field $Q \in(\operatorname{Vec}(X))_{\overline{1}}$ we define the odd flux map

$$
g_{Q}[S]: \operatorname{Map}(S, X) \longrightarrow \operatorname{Map}\left(S \times \mathbf{R}^{0 \mid 1}, X\right)
$$

and an even vector field $\iota_{Q} \in(\operatorname{Vec}(\Pi T X))_{\overline{0}}$ with the flux:

$$
\begin{aligned}
& \exp \left(\iota_{Q}\right)[S]: \operatorname{Map}\left(S \times \mathbf{R}^{0 \mid 1}, X\right) \longrightarrow \operatorname{Map}\left(S \times \mathbf{R}^{0 \mid 1}, X\right) \\
& \exp \left(\iota_{Q}\right)[S]=\mu[S] \circ g_{Q}\left[S \times \mathbf{R}^{0 \mid 1}\right]
\end{aligned}
$$

And $l_{Q}=\left[\iota_{Q}, d\right]$.

If we denote the coordinates of $\Pi T \Pi T X$ as $x, d x, D x, D d x$, the projection $\mu$ is:

$$
(f \circ \mu)(x, d x, D x, D d x)=f(x, d x+D x)
$$

\section{Submanifolds in $\Pi T X$}

Consider a submanifold $C \subset \Pi T X$ which is closed under $d$. (This means that the ideal generated by PDFs vanishing on $C \subset \Pi T X$ is closed under $d$.) We explained in section 2.2 that the action of the group $\Pi T G$ on $\Pi T X$ can be obtained from the action of $G$ on $X$. This, however, would not work for us here, because the resulting action of ПTG would not preserve $C \subset \Pi T X$. But in fact, the validity of eqs. (2.5) and (2.6) does not depend on how we constructed the action of $\Pi T G$ on $\Pi T X$. For any action of $\Pi T G$ on $\Pi T X$, commuting with the action of $d$ in the sense of eq. (2.12), the transformation $\omega \mapsto \Gamma \omega$ defined by eq. (2.4) will satisfy eqs. (2.5) and (2.6). This is equivalent to defining the structure of a differential $\mathbf{g}$-module ${ }^{2}$ on the space of functions on $C$. We will define the action of $C \mathbf{g}$ on $\Pi T X$ compatible with $d$ such that the vector fields representing $C \mathbf{g}$ will be tangent to $C \subset \Pi T X$. This defines the structure of a differential g-module on $\operatorname{Fun}(C)$. Then, we will define $\Gamma$ by eq. (2.4).

\section{Pure spinor target space as a subspace in $\Pi T X$}

\subsection{Supersymmetry generators and invariant derivatives}

It is always possible to find coordinates $x, \theta$ such that the supersymmetry generators have the form:

$$
q_{\alpha}^{L}=\frac{\partial}{\partial \theta_{L}^{\alpha}}-\left(\theta_{L} \gamma^{m}\right)_{\alpha} \frac{\partial}{\partial x^{m}}, \quad q_{\alpha}^{R}=\frac{\partial}{\partial \theta_{R}^{\alpha}}-\left(\theta_{R} \gamma^{m}\right)_{\alpha} \frac{\partial}{\partial x^{m}}
$$

To construct supersymmetry-invariant objects, it is useful to know the vector fields commuting with $q^{L \mid R}$. Besides translations $\frac{\partial}{\partial x^{m}}$, there are fermionic vector fields commuting with $q^{L \mid R}$. They are:

$$
\nabla_{\alpha}^{L}=\frac{\partial}{\partial \theta_{L}^{\alpha}}+\left(\theta_{L} \gamma^{m}\right)_{\alpha} \frac{\partial}{\partial x^{m}}, \quad \nabla_{\alpha}^{R}=\frac{\partial}{\partial \theta_{R}^{\alpha}}+\left(\theta_{R} \gamma^{m}\right)_{\alpha} \frac{\partial}{\partial x^{m}}
$$

\footnotetext{
${ }^{2}$ A differential $\mathbf{g}$-module is a representation $V$ of $C \mathbf{g}$, equipped with a differential $d_{V}$, compatible with the differential $d$ of $C \mathbf{g}$.
} 
The only non-zero commutators are

$$
\begin{aligned}
{\left[\nabla_{\alpha}^{L}, \nabla_{\beta}^{L}\right] } & =\left[\nabla_{\alpha}^{R}, \nabla_{\beta}^{R}\right]=2 \gamma_{\alpha \beta}^{m} \frac{\partial}{\partial x^{m}} \\
{\left[q_{\alpha}^{L}, q_{\beta}^{L}\right] } & =\left[q_{\alpha}^{R}, q_{\beta}^{R}\right]=-2 \gamma_{\alpha \beta}^{m} \frac{\partial}{\partial x^{m}}
\end{aligned}
$$

\subsection{Description of $C \subset \Pi T X$}

Consider the space $X$ parametrized by the coordinates $\left(x^{m}, \theta_{L}^{\alpha}, \theta_{R}^{\alpha}\right)$. Then $\Pi T X$ is parametrized by the coordinates $\left(x^{m}, \theta_{L}^{\alpha}, \theta_{R}^{\alpha}, d x^{m}, d \theta_{L}^{\alpha}, d \theta_{R}^{\alpha}\right)$ where " $d x "$ ", " $d \theta^{\prime}$ are considered one letter. Then, to describe the pure spinor string in a flat background, we choose as a target the subspace of $C \subset \Pi T X$ defined by the following conditions

$$
\begin{array}{r}
d \theta_{L} \gamma^{m} d \theta_{L}=d \theta_{R} \gamma^{m} d \theta_{R}=0 \\
d x^{m}-d \theta_{L} \gamma^{m} \theta_{L}-d \theta_{R} \gamma^{m} \theta_{R}=0
\end{array}
$$

The constraint defined by eq. (4.5) is the pure spinor constraint, it is essentially postulated. The constraint of eq. (4.6) is characterized by:

$$
{ }^{\iota} \nabla^{L \mid R}\left(d x^{m}-d \theta_{L} \gamma^{m} \theta_{L}-d \theta_{R} \gamma^{m} \theta_{R}\right)=0
$$

where $\nabla^{L \mid R}$ are defined by eq. (4.2).

The main properties of the ideal generated by eqs. (4.5) and (4.6) are:

1. It is $q^{L}$ - and $q^{R}$-invariant, i.e. $\mathcal{L}_{q^{L \mid R}}$ annihilate ${ }^{3} d \theta_{L} \gamma^{m} d \theta_{L}, d \theta_{R} \gamma^{m} d \theta_{R}$, and $d x^{m}-$ $d \theta_{L} \gamma^{m} \theta_{L}-d \theta_{R} \gamma^{m} \theta_{R}$

2. It is $d$-closed.

Usually one denotes:

$$
\lambda_{L}^{\alpha}=d \theta_{L}^{\alpha}, \quad \lambda_{R}^{\alpha}=d \theta_{R}^{\alpha}
$$

The BRST operator $Q$ is just $d$ :

$$
\begin{aligned}
Q\left(x^{m}\right) & =\left(\lambda_{L} \gamma^{m} \theta_{L}\right)+\left(\lambda_{R} \gamma^{m} \theta_{R}\right) \\
Q\left(\theta_{L}^{\alpha}\right) & =\lambda_{L}^{\alpha} \\
Q\left(\theta_{R}^{\alpha}\right) & =\lambda_{R}^{\alpha} \\
Q\left(\lambda_{L}^{\alpha}\right) & =0 \\
Q\left(\lambda_{R}^{\alpha}\right) & =0
\end{aligned}
$$

Eq. (4.7) is promising for constructing the action of $\Pi T G$ on $\Pi T X$ as described in section 3 . We also observe:

$$
\mathcal{L}_{\nabla^{L \mid R}}\left(d \theta_{L \mid R} \gamma^{m} d \theta_{L \mid R}\right)=0
$$

However, let us keep in mind that:

$$
\begin{array}{r}
{ }_{\nabla^{L} \mid R}\left(d \theta_{L \mid R} \gamma^{m} d \theta_{L \mid R}\right) \neq 0 \\
\mathcal{L}_{\nabla^{L \mid R}}\left(d x^{m}-d \theta_{L} \gamma^{m} \theta_{L}-d \theta_{R} \gamma^{m} \theta_{R}\right) \neq 0
\end{array}
$$

\footnotetext{
${ }^{3}$ To see the vanishing of $\mathcal{L}_{q L \mid R}\left(d x^{m}-d \theta_{L} \gamma^{m} \theta_{L}-d \theta_{R} \gamma^{m} \theta_{R}\right)=0$, we first observe that this expression does not contain $d x$. But then, it cannot contain neither $d \theta_{L}$ nor $d \theta_{R}$ because of eq. (4.7).
} 


\subsection{Solving the pure spinor constraint}

The ten-dimensional gamma matrices can be decomposed to give the eight-dimensional ones and also chiral projectors

$$
\begin{array}{rlrl}
{\left[\gamma_{\alpha \beta}^{i}\right]} & =\left(\begin{array}{cc}
0 & \sigma_{a \dot{b}}^{i} \\
\sigma_{\dot{a} b}^{i} & 0
\end{array}\right), & & {\left[\gamma^{i \alpha \beta}\right]=\left(\begin{array}{cc}
0 & \sigma^{i a \dot{b}} \\
\sigma^{i \dot{a} b} & 0
\end{array}\right)} \\
{\left[\gamma_{\alpha \beta}^{+}\right]=\left(\begin{array}{cc}
0 & 0 \\
0 & -\delta_{\dot{a} \dot{b}}
\end{array}\right),} & {\left[\gamma^{+\alpha \beta}\right]=\left(\begin{array}{cc}
\delta^{a b} & 0 \\
0 & 0
\end{array}\right)} \\
{\left[\gamma_{\alpha \beta}^{-}\right]=\left(\begin{array}{cc}
-\delta_{a b} & 0 \\
0 & 0
\end{array}\right),} & {\left[\gamma^{-\alpha \beta}\right]=\left(\begin{array}{cc}
0 & 0 \\
0 & \delta^{\dot{a} \dot{b}}
\end{array}\right)}
\end{array}
$$

where $i, j, a, b, \dot{a}, \dot{b}=1, \ldots, 8$. Then, we will use the Kronecker deltas $\delta_{a b}, \delta_{\dot{a} \dot{b}}, \delta^{a b}, \delta^{\dot{a} \dot{b}}$ to raise and lower spinor indices. The Pauli matrices are such that $\sigma_{a \dot{b}}^{i}=\sigma_{\dot{b} a}^{i}$ and $\left(\sigma^{j}\right)^{\dot{a} b}=\delta^{\dot{a} \dot{b}} \delta^{b a} \sigma_{a \dot{b}}^{j}$. These matrices satisfy

$$
\begin{aligned}
\left(\sigma^{i}\right)_{a \dot{b}}\left(\sigma^{j}\right)^{\dot{b} c}+\left(\sigma^{j}\right)_{a \dot{b}}\left(\sigma^{i}\right)^{\dot{b} c} & =2 \delta_{a}^{c} \delta^{i j} \\
\left(\sigma^{i}\right)_{a \dot{b}}\left(\sigma_{i}\right)_{c \dot{d}}+\left(\sigma^{i}\right)_{a \dot{d}}\left(\sigma_{i}\right)_{\dot{b} c} & =2 \delta_{a c} \delta_{\dot{d} \dot{b}} \\
\left(\sigma^{i j}\right)^{a}{ }_{b}\left(\sigma_{i j}\right)^{c}{ }_{d} & =8 \delta^{a c} \delta_{b d}-8 \delta_{d}^{a} \delta_{b}^{c} \\
\left(\sigma^{i j}\right)^{a}{ }_{b}\left(\sigma_{i j}\right)_{\dot{d}}^{\dot{c}} & =4\left(\sigma^{i}\right)^{a \dot{c}}\left(\sigma_{i}\right)_{b \dot{d}}-4 \delta_{b}^{a} \delta_{\dot{d}}^{\dot{c}}
\end{aligned}
$$

In $\mathrm{SO}(8)$ components, the pure spinor constraints for $\lambda^{\alpha}=\left(\lambda^{a}, \bar{\lambda}^{\dot{a}}\right)$ are:

$$
\lambda^{a} \delta_{a b} \lambda^{b}=0, \quad \bar{\lambda}^{\dot{a}} \delta_{\dot{a} \dot{b}} \bar{\lambda}^{\dot{b}}=0, \quad \lambda^{a} \sigma_{a \dot{a}}^{i} \bar{\lambda}^{\dot{a}}=0
$$

Both $\lambda_{L}$ and $\lambda_{R}$ satisfy these constraints.

They can be solved as follows. Let us define:

$$
\lambda_{ \pm}^{a}:=\frac{1}{2}\left(\lambda_{L}^{a} \pm i \lambda_{R}^{a}\right), \quad \bar{\lambda}_{ \pm}^{\dot{a}}:=\frac{1}{2}\left(\bar{\lambda}_{L}^{\dot{a}} \pm i \bar{\lambda}_{R}^{\dot{a}}\right)
$$

Eqs. (4.5) imply:

$$
\begin{aligned}
& \lambda_{+}^{2}+\lambda_{-}^{2}=\lambda_{+} \lambda_{-}=0 \\
& \bar{\lambda}_{+}^{2}+\bar{\lambda}_{-}^{2}=\bar{\lambda}_{+} \bar{\lambda}_{-}=0 \\
& \lambda_{+} \sigma^{i} \bar{\lambda}_{+}+\lambda_{-} \sigma^{i} \bar{\lambda}_{-}=\lambda_{+} \sigma^{i} \bar{\lambda}_{-}+\lambda_{-} \sigma^{i} \bar{\lambda}_{+}=0
\end{aligned}
$$

We can solve these equations for $\lambda_{-}^{a}$ in terms of the rest of variables:

$$
\lambda_{-}^{a}=-\frac{\left(\bar{\lambda}_{+} \sigma_{i j} \bar{\lambda}_{-}\right)}{4\left(\bar{\lambda}_{+} \bar{\lambda}_{+}\right)}\left(\sigma^{i j}\right)^{a}{ }_{b} \lambda_{+}^{b}=\frac{\left(\bar{\lambda}_{L} \sigma^{i j} \bar{\lambda}_{R}\right)}{4\left(\bar{\lambda}_{L} \bar{\lambda}_{R}\right)}\left(\sigma_{i j}\right)^{a}{ }_{b} \lambda_{+}^{b}
$$

where $\lambda_{+}^{a}$ is unconstrained and $\left(\bar{\lambda}_{L}^{\dot{a}}, \bar{\lambda}_{R}^{\dot{a}}\right)$ are still subject to the conditions $\left(\bar{\lambda}_{L}\right)^{2}=\left(\bar{\lambda}_{R}\right)^{2}=$ 0 . Let us introduce the $8 \times 8$ matrix $M$ :

$$
M_{b}^{a}=\frac{\partial \lambda_{-}^{a}}{\partial \lambda_{+}^{b}}=\frac{\left(\bar{\lambda}_{L} \sigma^{i j} \bar{\lambda}_{R}\right)}{4\left(\bar{\lambda}_{L} \bar{\lambda}_{R}\right)}\left(\sigma_{i j}\right)_{b}^{a}
$$


This matrix inherits the antisymmetry properties of the Lorentz generators $\left(\sigma_{i j}\right)^{a}{ }_{b}$ when their indices are raised and lowered with Kronecker deltas

$$
M_{a b}=-M_{b a} \quad \text { equivalently } \quad M_{b}^{a}=-M_{b}^{a}
$$

and it squares to identity:

$$
M^{a}{ }_{b} M^{b}{ }_{c}=\delta_{c}^{a}
$$

(Eq. (4.26) follows from considering separately the identity $\left(\lambda_{+}\right)^{2}+\left(\lambda_{-}\right)^{2}=0$.)

In the light-cone coordinates the conditions (4.6) are written as

$$
\begin{aligned}
d x^{+}-2\left(\lambda_{+} \theta_{-}+\lambda_{-} \theta_{+}\right) & =0 \\
d x^{-}-2\left(\bar{\lambda}_{+} \bar{\theta}_{-}+\bar{\lambda}_{-} \bar{\theta}_{+}\right) & =0 \\
d x^{i}-2\left(\lambda_{+} \sigma^{i} \bar{\theta}_{-}+\lambda_{-} \sigma^{i} \bar{\theta}_{+}+\bar{\lambda}_{+} \sigma^{i} \theta_{-}+\bar{\lambda}_{-} \sigma^{i} \theta_{+}\right) & =0
\end{aligned}
$$

Consider the subspace of functions which only depend on $x^{+}$and do not on $x^{-}$nor $x^{i}$. On this subspace:

$$
\begin{aligned}
\nabla_{a}^{ \pm}=\frac{\partial}{\partial \theta_{ \pm}^{a}}+2 \theta_{\mp a} \frac{\partial}{\partial x^{+}}, & \bar{\nabla}_{\dot{a}}^{ \pm}=\frac{\partial}{\partial \bar{\theta}_{ \pm}^{\dot{a}}} \\
q_{a}^{ \pm}=\frac{\partial}{\partial \theta_{ \pm}^{a}}-2 \theta_{\mp a} \frac{\partial}{\partial x^{+}}, & \bar{q}_{\dot{a}}^{ \pm}=\frac{\partial}{\partial \bar{\theta}_{ \pm}^{\dot{a}}}
\end{aligned}
$$

while the only non-zero commutators will be $\left[\nabla_{a}^{+}, \nabla_{b}^{-}\right]=-\left[q_{a}^{+}, q_{b}^{-}\right]=4 \delta_{a b} \partial_{+}$.

\section{$5 \quad$ An action of $C \mathrm{R}^{0 \mid 8}$ on $C$}

Let us consider the coordinates on the fiber of $C:\left(\lambda_{+}^{a}, \bar{\lambda}_{+}^{a}, \bar{\lambda}_{-}^{a}\right)$. Let us introduce the following vector fields on $C$ :

$$
i_{a}=\frac{\partial}{\partial \lambda_{+}^{a}}
$$

These $i_{a}$ are vertical vector fields (tangent to the fiber). They commute: $\left[i_{a}, i_{b}\right]=0$. Equivalently, we can start with unconstrained $\lambda$, and define:

$$
i_{a}=\frac{\partial}{\partial \lambda_{+}^{a}}+\left.\frac{\partial \lambda_{-}^{b}}{\partial \lambda_{+}^{a}}\right|_{\bar{\lambda}=\mathrm{const}} \frac{\partial}{\partial \lambda_{-}^{b}}+\left.\frac{\partial d x^{m}}{\partial \lambda_{+}^{a}}\right|_{\bar{\lambda}=\mathrm{const}} \frac{\partial}{\partial d x^{m}}+\left.\frac{\partial d x^{+}}{\partial \lambda_{+}^{a}}\right|_{\bar{\lambda}=\mathrm{const}} \frac{\partial}{\partial d x^{+}}
$$

where the derivative $\left.\frac{\partial \lambda_{-}^{b}}{\partial \lambda_{+}^{a}}\right|_{\bar{\lambda}=\text { const }}$ is of the r.h.s. of eq. (4.23), and $\frac{\partial d x}{\partial \lambda_{+}^{a}}$ includes the explicit dependence of $d x$ on $\lambda_{+}$as well as dependence through $\lambda_{-}\left(\lambda_{+}\right)$(see eqs. (4.27), (4.28) and (4.29)). This vector field is tangent to the cone. This is the same as to consider the vector field of eq. (5.1) on $C$ in coordinates $\left(\lambda_{+}^{a}, \bar{\lambda}_{+}^{a}, \bar{\lambda}_{-}^{a}\right)$.

Next, we define:

$$
l_{a}=\left[i_{a}, d\right]=\Pi T\left(\nabla_{a}^{+}\right)+\left.\frac{\partial \lambda_{-}^{b}}{\partial \lambda_{+}^{a}}\right|_{\bar{\lambda}=\text { const }} \Pi T\left(\nabla_{b}^{-}\right)
$$


In deriving eq. (5.3), the following observation is useful. If $v$ is an even vertical vector field on $\Pi T X$, then:

$$
\mu_{*}\left(\iota_{\Pi T \Pi T X}\left(\left[v, d_{\Pi T X}\right]\right)\right)=v
$$

where $\mu$ is from eq. (2.16). Indeed, the r.h.s. of eq. (5.3) is fixed by $\mu_{*}\left(\iota_{\Pi T \Pi T X} l_{a}\right)$ being as in eq. (5.2) (this follows from the definition of $\nabla$, eq. (4.7)).

We observe that $\lambda_{-}^{b}$ is a linear function of $\lambda_{+}^{a}$, thus $\frac{\partial^{2} \lambda_{-}^{a}}{\partial \lambda_{+}^{b} \partial \lambda_{+}^{c}}=0$. Also $\frac{\partial \lambda_{-}^{b}}{\partial \lambda_{+}^{a}}+\frac{\partial \lambda_{-}^{a}}{\partial \lambda_{+}^{b}}=0$. Therefore:

$$
\left[l_{a}, i_{b}\right]=0
$$

and this implies

$$
\left[l_{a}, l_{b}\right]=0
$$

This means that $d, i_{a}, l_{b}$ define an action of the differential Lie superalgebra $C \mathbf{R}^{0 \mid 8}$ on $C$.

\subsection{Type IIB supergravity vertex operator at picture $(-8)$}

In coordinates $y^{+}=x^{+}-2 \theta_{+}^{a} \theta_{-a}$ :

$$
\begin{aligned}
\nabla_{+a} & =\frac{\partial}{\partial \theta_{-}^{a}}+4 \theta_{+a} \frac{\partial}{\partial y^{+}}, & \nabla_{-a} & =\frac{\partial}{\partial \theta_{+}^{a}} \\
q_{+a} & =\frac{\partial}{\partial \theta_{-}^{a}}, & q_{-a} & =\frac{\partial}{\partial \theta_{+}^{a}}-4 \theta_{-a} \frac{\partial}{\partial y^{+}}
\end{aligned}
$$

We have lowered their,+- superscripts such that $\nabla_{ \pm a}:=\nabla_{a}^{\mp}$ and $q_{ \pm a}:=q_{a}^{\mp}$. These coordinates simplify the $\theta$-expansion of the dilaton superfield $\Phi\left(y^{+}, \theta_{ \pm}\right)$since it satisfies $\nabla_{-} \Phi=0$. We have

$$
\begin{aligned}
& \Phi\left(y^{+}, \theta_{-}\right)= \\
& =e^{i k_{+} y^{+}}\left(C+C^{a_{1}} \theta_{-a_{1}}+\frac{1}{2 !} C^{a_{1} a_{2}} \theta_{-a_{1}} \theta_{-a_{2}}+\ldots+\frac{1}{8 !} C^{a_{1} \ldots a_{8}} \theta_{-a_{1}} \ldots \theta_{-a_{8}}\right)
\end{aligned}
$$

where the constants $C^{a_{1} \ldots a_{k}}$ are bosonic (fermionic) when $k$ is even (odd).

When working with the vertex operator corresponding to type IIB supergravity is useful to know the following identity

$$
\begin{aligned}
& \prod_{b=1}^{8} \nabla_{+b}\left(e^{i k_{+} y^{+}} \theta_{-}^{a_{1}} \ldots \theta_{-}^{a_{k}} C_{a_{1} \ldots a_{k}}\right)= \\
& =\left(4 \partial_{+}\right)^{8-k} C^{a_{1} \ldots a_{k}} q_{-a_{1}} \ldots q_{-a_{k}}\left(e^{i k_{+} y^{+}} \prod_{b=1}^{8} \theta_{+}^{b}\right)
\end{aligned}
$$

which in turn implies that

$$
\begin{aligned}
& \frac{1}{\left(4 \partial_{+}\right)^{8}} \prod_{b=1}^{8} \nabla_{+}^{b} \Phi\left(y^{+}, \theta_{-}\right)= \\
& =\left[\sum_{k=0}^{8} \frac{\left(4 \partial_{+}\right)^{-k}}{k !} C^{a_{1} \ldots a_{k}} q_{-a_{1}} \ldots q_{-a_{k}}\right]\left(e^{i k_{+} y^{+}} \prod_{b=1}^{8} \theta_{+}^{b}\right)
\end{aligned}
$$


We start by considering the vertex operator in a (-8)-picture that corresponds to the type IIB supergravity scalar state

$$
V_{-8}^{\text {scalar }}=\left(\bar{\lambda}_{L} \bar{\lambda}_{R}\right) e^{i k_{+} y^{+}} \prod_{a=1}^{8} \theta_{+}^{a} \delta\left(\lambda_{+}^{a}\right)
$$

and by applying the $q_{-a}$ supersymmetry generators, we can generate the full type IIB supergravity multiplet using (5.11)

$$
V_{-8}=\left(\bar{\lambda}_{L} \bar{\lambda}_{R}\right) \frac{1}{\left(4 \partial_{+}\right)^{8}}\left(\prod_{a=1}^{8} \delta\left(\lambda_{+}^{a}\right) \nabla_{+}^{a}\right) \Phi\left(y^{+}, \theta_{-}\right)
$$

\subsection{Type IIB supergravity vertex operator at picture $(0)$}

We will now use the vector fields $\rho\left(l_{a}\right), \rho\left(i_{a}\right)$ constructed in section 5 to transform the supergravity vertex from picture -8 to picture 0 . As we explained in section 2.2 and section 3, this amounts to computing the integral:

$$
V_{0}:=\Gamma V_{-8}=\int \prod_{a=1}^{8} D\left(d \epsilon^{a}\right) \frac{\partial}{\partial \epsilon^{a}} \exp \left(\epsilon^{a} \rho\left(l_{a}\right)+d \epsilon^{a} \rho\left(i_{a}\right)\right) V_{-8}
$$

The explicit expressions for these vector fields are:

$$
\begin{aligned}
& \rho\left(l_{a}\right)=\Pi T\left(\nabla_{a}^{+}\right)+\frac{\partial \lambda_{-}^{b}}{\partial \lambda_{+}^{a}} \Pi T\left(\nabla_{b}^{-}\right)=\left(\nabla_{a}^{+}-2 \lambda_{-a} \frac{\partial}{\partial d x^{+}}\right)+\frac{\partial \lambda_{-}^{b}}{\partial \lambda_{+}^{a}}\left(\nabla_{b}^{-}-2 \lambda_{+b} \frac{\partial}{\partial d x^{+}}\right) \\
& \rho\left(i_{a}\right)=\left(\frac{\partial}{\partial \lambda_{+}^{a}}+2 \theta_{-}^{a} \frac{\partial}{\partial d x^{+}}\right)+\frac{\partial \lambda_{-}^{b}}{\partial \lambda_{+}^{a}}\left(\frac{\partial}{\partial \lambda_{-}^{b}}+2 \theta_{+}^{b} \frac{\partial}{\partial d x^{+}}\right) \\
& \rho(d)=d
\end{aligned}
$$

After some computation we can verify that these vector fields satisfy

$$
\left[\rho\left(l_{a}\right), \rho\left(l_{b}\right)\right]=\left[\rho\left(i_{a}\right), \rho\left(i_{b}\right)\right]=\left[\rho\left(l_{a}\right), \rho\left(i_{b}\right)\right]=0
$$

Now we can integrate along the orbits of $\Pi T \mathbf{R}^{0 \mid 8}$, as in eq. (2.14):

$$
\begin{aligned}
V_{0}:=\Gamma V_{-8} & =\int \prod_{a=1}^{8} D\left(d \epsilon^{a}\right) \frac{\partial}{\partial \epsilon^{a}} \exp \left(\epsilon^{a} \rho\left(l_{a}\right)+d \epsilon^{a} \rho\left(i_{a}\right)\right) V_{-8}= \\
& =\int \prod_{a=1}^{8} D\left(d \epsilon^{a}\right) \rho\left(l_{1}\right) \ldots \rho\left(l_{8}\right) \exp \left(d \epsilon^{a} \rho\left(i_{a}\right)\right) V_{-8}= \\
& =\frac{1}{\left(4 \partial_{+}\right)^{8}}\left(\bar{\lambda}_{L} \bar{\lambda}_{R}\right) \rho\left(l_{1}\right) \ldots \rho\left(l_{8}\right)\left(\prod_{a=1}^{8} \nabla_{+}^{a}\right) \Phi\left(x^{+}-2 \theta_{+}^{a} \theta_{-a}, \theta_{-}\right)
\end{aligned}
$$

where in the last line we have used that the only $\lambda_{ \pm}$-dependence of $V_{-8}$ is through $\delta\left(\lambda_{+}^{a}\right)$ which means that the integral on $d \epsilon$ eliminates all deltas at once. To proceed with the 
computation first notice that there is no dependence on coordinate $d x^{+}$, so we can drop the $\frac{\partial}{\partial d x^{+}}$-part in $\rho\left(l_{a}\right)$. We also change to coordinates $y^{+}=x^{+}-2 \theta_{+}^{a} \theta_{-a}$ to obtain

$$
\begin{aligned}
\Gamma V_{-8}= & \frac{1}{\left(4 \partial_{+}\right)^{8}}\left(\bar{\lambda}_{L} \bar{\lambda}_{R}\right) \prod_{a=1}^{8}\left(\nabla_{a}^{+}+\frac{\partial \lambda_{-}^{b}}{\partial \lambda_{+}^{a}} \nabla_{b}^{-}\right)\left(\prod_{b=1}^{8} \nabla_{+}^{b}\right) \Phi\left(y^{+}, \theta_{-}\right) \\
= & \frac{1}{\left(4 \partial_{+}\right)^{8}}\left(\bar{\lambda}_{L} \bar{\lambda}_{R}\right) \epsilon^{a_{1} \ldots a_{8}}\left[\frac{1}{8 !} \nabla_{a_{1}}^{+} \ldots \nabla_{a_{8}}^{+}+\frac{\left(4 \partial_{+}\right)}{6 ! 2 !} \nabla_{a_{1}}^{+} \ldots \nabla_{a_{6}}^{+} \frac{\partial \lambda_{-a_{8}}}{\partial \lambda_{+}^{a_{7}}}\right. \\
& +\frac{\left(4 \partial_{+}\right)^{2}}{4 !(2 !)^{3}} \nabla_{a_{1}}^{+} \nabla_{a_{2}}^{+} \nabla_{a_{3}}^{+} \nabla_{a_{4}}^{+} \frac{\partial \lambda_{-a_{6}}}{\partial \lambda_{+}^{a_{5}}} \frac{\partial \lambda_{-a_{8}}}{\partial \lambda_{+}^{a_{7}}}+\frac{\left(4 \partial_{+}\right)^{3}}{3 !(2 !)^{4}} \nabla_{a_{1}}^{+} \nabla_{a_{2}}^{+} \frac{\partial \lambda_{-a_{4}}}{\partial \lambda_{+}^{a_{3}}} \frac{\partial \lambda_{-a_{6}}}{\partial \lambda_{+}^{a_{5}}} \frac{\partial \lambda_{-a_{8}}}{\partial \lambda_{+}^{a_{7}}} \\
& \left.+\frac{\left(4 \partial_{+}\right)^{4}}{4 !(2 !)^{4}} \frac{\partial \lambda_{-a_{2}}}{\partial \lambda_{+}^{a_{1}}} \frac{\partial \lambda_{-a_{4}}}{\partial \lambda_{+}^{a_{3}}} \frac{\partial \lambda_{-a_{6}}}{\partial \lambda_{+}^{a_{5}}} \frac{\partial \lambda_{-a_{8}}}{\partial \lambda_{+}^{a_{7}}}\right]\left(\prod_{b=1}^{8} \nabla_{+}^{b}\right) \Phi\left(y^{+}, \theta_{-}\right)
\end{aligned}
$$

We want to further transform this expression. First, we substitute eq. (4.23) for $\frac{\partial \lambda_{-}}{\partial \lambda_{+}}$. Then, we anticommute all $\nabla^{+}$'s all the way to right and make use of:

$$
\nabla^{+} \Phi=0
$$

(Also, remember that $\nabla_{a}^{-}=\nabla_{+a}$ and $\nabla_{a}^{+}=\nabla_{-a}$ ). The computation uses some identities for the commutators of the SUSY-invariant derivatives, namely eqs. (A.5), (A.6) and (A.7) in appendix section A. The result is the following expression for the type IIB supergravity vertex operator $V_{0}=\Gamma V_{-8}$ :

$$
\begin{aligned}
V_{0}= & \left(\bar{\lambda}_{L} \bar{\lambda}_{R}\right)\left[\Phi+\frac{1}{\left(32 \partial_{+}\right)} \frac{\left(\bar{\lambda}_{L} \sigma^{i j} \bar{\lambda}_{R}\right)}{\left(\bar{\lambda}_{L} \bar{\lambda}_{R}\right)}\left(\nabla_{+} \sigma_{i j} \nabla_{+}\right) \Phi\right. \\
& +\frac{1}{2 !\left(32 \partial_{+}\right)^{2}} \prod_{n=1}^{2} \frac{\left(\bar{\lambda}_{L} \sigma^{i_{n} j_{n}} \bar{\lambda}_{R}\right)}{\left(\bar{\lambda}_{L} \bar{\lambda}_{R}\right)}\left(\nabla_{+} \sigma_{i_{n} j_{n}} \nabla_{+}\right) \Phi \\
& +\frac{1}{3 !\left(32 \partial_{+}\right)^{3}} \prod_{n=1}^{3} \frac{\left(\bar{\lambda}_{L} \sigma^{i_{n} j_{n}} \bar{\lambda}_{R}\right)}{\left(\bar{\lambda}_{L} \bar{\lambda}_{R}\right)}\left(\nabla_{+} \sigma_{i_{n} j_{n}} \nabla_{+}\right) \Phi \\
& \left.+\frac{1}{4 !\left(32 \partial_{+}\right)^{4}} \prod_{n=1}^{4} \frac{\left(\bar{\lambda}_{L} \sigma^{i_{n} j_{n}} \bar{\lambda}_{R}\right)}{\left(\bar{\lambda}_{L} \bar{\lambda}_{R}\right)}\left(\nabla_{+} \sigma_{i_{n} j_{n}} \nabla_{+}\right) \Phi\right]
\end{aligned}
$$

This formula reproduces the result of [10] where the computation was performed using the PCOs in their standard form $\Gamma_{a}=\left[Q_{B R S T}, \Theta\left(\omega_{a}^{+}\right)\right]$. Our computation is, in some sense, more streamlined. In particular, we do not have any poles in $\lambda_{+}^{a}$. (The computation of [10] has such poles at the middle steps; they appear each time a $\Theta\left(\omega_{a}^{+}\right)$hit a $\delta\left(\lambda_{+}^{a}\right)$.)

Still, our computation does suffer from the poles with denominators $\left(\bar{\lambda}_{L} \bar{\lambda}_{R}\right)$. The only terms which could potentially have such denominators are the third, fourth and fifth. Notice, however, that the fifth term is $\left(\bar{\lambda}_{L} \bar{\lambda}_{R}\right)$ times an expression which contains $\bar{\lambda}_{L}$ and $\bar{\lambda}_{R}$ only through the Pfaffian of the matrix $M$ defined in eq. (4.24). Since $M^{2}=\mathbf{1}$, the Pfaffian is \pm 1 . Therefore, this last term only depends on $\bar{\lambda}_{L}$ and $\bar{\lambda}_{R}$ through the factor $\left(\bar{\lambda}_{L} \bar{\lambda}_{R}\right)$. It is equal to:

$$
\pm\left(\bar{\lambda}_{L} \bar{\lambda}_{R}\right) \frac{1}{\left(16 \partial_{+}\right)^{4}} \nabla_{+}^{1} \nabla_{+}^{2} \cdots \nabla_{+}^{8} \Phi
$$


The fourth term (the one containing six $\nabla_{+}$) is also nonsingular. Indeed, it follows from $\operatorname{det}(M)=1$ that its dependence on $\bar{\lambda}_{L}$ and $\bar{\lambda}_{R}$ is linear in $\left(\bar{\lambda}_{L} \bar{\lambda}_{R}\right) M$. The only term which could potentially have a pole is the third (middle) term, which contains four $\nabla_{+}$. It could have a first order pole in $\left(\bar{\lambda}_{L} \bar{\lambda}_{R}\right)$. The cancellation of this pole is not obvious; it was proven in [10] using certain identities for the $\left(\sigma^{j}\right)_{a \dot{a}}$ matrices.

Thus the zero-picture vertex operator is actually of the form $V_{0}=\bar{\lambda}_{L}^{\dot{a}} \bar{\lambda}_{R}^{\dot{b}} A_{\dot{a} \dot{b}}\left(x^{+}, \theta_{ \pm}\right)$.

\section{Open questions}

1. We constructed some action of $C \mathbf{R}^{0 \mid 8}$ on the pure spinor target space. How flexible is this construction? Is it in some sense natural? Given $V_{-8}$, is there a canonical way to construct $V_{0}$ ? (This question is very important for the computation of amplitudes. If there is no canonical map $V_{-8} \rightarrow V_{0}$, then using the -8 picture in computation of amplitudes is not apriori justified.)

2. It is not immediately clear how to apply our method in AdS. A naive analogue of our vector fields $i_{a}$ and $l_{a}$ from section 5 is not well-defined in AdS (it would not be gauge invariant).

3. The use of delta-functions on the cone is potentially dangerous. We would want to understand, when generalized functions of the form of products of $\delta(\lambda)$ are welldefined.

\section{A Anticommuting $\nabla_{-}$and $\nabla_{+}$}

Here we will prove some formulas of SUSY-invariant derivative $\nabla$ which are needed to derive eq. (5.22) from eq. (5.20).

There are two relations that are useful when anticommuting the $\nabla_{-}$and $\nabla_{+}$operators. The first one which can be proven by induction is

$$
\begin{aligned}
\nabla_{-a_{k}} \ldots \nabla_{-a_{1}} \nabla_{+}^{a_{1}} \ldots \nabla_{+}^{a_{k}}= & \left(\nabla_{-a_{k}} \nabla_{+}^{a_{k}}\right) \nabla_{-a_{k-1}} \ldots \nabla_{-a_{1}} \nabla_{+}^{a_{1}} \ldots \nabla_{+}^{a_{k-1}} \\
& -(k-1)\left(4 \partial_{+}\right) \nabla_{-a_{k-1}} \ldots \nabla_{-a_{1}} \nabla_{+}^{a_{1}} \ldots \nabla_{+}^{a_{k-1}}
\end{aligned}
$$

This equation is used to prove the second one

$$
\begin{aligned}
& \nabla_{-a_{n}} \ldots \nabla_{-a_{1}} \nabla_{+}^{a_{1}} \ldots \nabla_{+}^{a_{n}}\left(\nabla_{+} \sigma^{k l} \nabla_{+}\right)= \\
& =\left(\nabla_{+} \sigma^{k l} \nabla_{+}\right)\left(\sum_{i=0}^{n} C_{i}^{(n)}\left(4 \partial_{+}\right)^{n-i} \nabla_{-a_{i}} \ldots \nabla_{-a_{1}} \nabla_{+}^{a_{1}} \ldots \nabla_{+}^{a_{i}}\right)
\end{aligned}
$$

where the numeric coefficients $C_{i}^{(n)}$ with $i=0, \ldots, n$ are found recursively starting with

$$
C_{0}^{(1)}=-2, \quad C_{1}^{(1)}=1
$$


and the following ones with

$$
\begin{aligned}
& C_{0}^{(n+1)}=-(n+2) C_{0}^{(n)} \\
& C_{i}^{(n+1)}=(i-n-2) C_{i}^{(n)}+C_{i-1}^{(n)}, \quad i=1, \ldots, n \\
& C_{n+1}^{(n+1)}=C_{n}^{(n)}
\end{aligned}
$$

Using equations (A.1) and (A.2) continuously we can finally arrive at the expressions needed to compute the picture-zero vertex operator

$$
\begin{array}{r}
\nabla_{-a_{2}} \nabla_{-a_{1}}\left(\nabla_{+} \sigma^{i j} \nabla_{+}\right)\left(\nabla_{+} \sigma^{k l} \nabla_{+}\right)\left(\nabla_{+} \sigma^{m n} \nabla_{+}\right) \nabla_{+}^{a_{1}} \nabla_{+}^{a_{2}} \Phi= \\
=2 !\left(4 \partial_{+}\right)^{2}\left(\nabla_{+} \sigma^{i j} \nabla_{+}\right)\left(\nabla_{+} \sigma^{k l} \nabla_{+}\right)\left(\nabla_{+} \sigma^{m n} \nabla_{+}\right) \Phi \\
\nabla_{-a_{4}} \ldots \nabla_{-a_{1}}\left(\nabla_{+} \sigma^{i j} \nabla_{+}\right)\left(\nabla_{+} \sigma^{k l} \nabla_{+}\right) \nabla_{+}^{a_{1}} \ldots \nabla_{+}^{a_{4}} \Phi= \\
=4 !\left(4 \partial_{+}\right)^{4}\left(\nabla_{+} \sigma^{i j} \nabla_{+}\right)\left(\nabla_{+} \sigma^{k l} \nabla_{+}\right) \Phi \\
\nabla_{-a_{6}} \ldots \nabla_{-a_{1}}\left(\nabla_{+} \sigma^{i j} \nabla_{+}\right) \nabla_{+}^{a_{1}} \ldots \nabla_{+}^{a_{6}} \Phi= \\
=6 !\left(4 \partial_{+}\right)^{6}\left(\nabla_{+} \sigma^{i j} \nabla_{+}\right) \Phi
\end{array}
$$

\section{Acknowledgments}

We want to thank Nathan Berkovits, Lucas Martins and Michael Movshev for discussions. This work was supported in part by FAPESP grant 2014/18634-9 "Dualidade Gravitação/Teoria de Gauge".

Open Access. This article is distributed under the terms of the Creative Commons Attribution License (CC-BY 4.0), which permits any use, distribution and reproduction in any medium, provided the original author(s) and source are credited.

\section{References}

[1] N. Berkovits, Simplifying and Extending the AdS $S_{5} \times S^{5}$ Pure Spinor Formalism, JHEP 09 (2009) 051 [arXiv:0812.5074] [INSPIRE].

[2] N. Berkovits, Perturbative Super-Yang-Mills from the Topological AdS $S_{5} \times S^{5} \sigma$-model, JHEP 09 (2008) 088 [arXiv:0806.1960] [INSPIRE].

[3] N. Berkovits and O. Chandía, Superstring vertex operators in an $A d S_{5} \times S^{5}$ background, Nucl. Phys. B 596 (2001) 185 [hep-th/0009168] [INSPIRE].

[4] A. Mikhailov, Symmetries of massless vertex operators in $A d S_{5} \times S^{5}$, J. Geom. Phys. 62 (2012) 479 [arXiv:0903.5022] [INSPIRE].

[5] O.A. Bedoya, L. Bevilaqua, A. Mikhailov and V.O. Rivelles, Notes on $\beta$-deformations of the pure spinor superstring in $A d S_{5} \times S^{5}$, Nucl. Phys. B 848 (2011) 155 [arXiv:1005.0049] [INSPIRE].

[6] N. Berkovits, Half-BPS vertex operators of the $A d S_{5} \times S^{5}$ superstring, JHEP 07 (2019) 084 [arXiv: 1904.06564] [INSPIRE]. 
[7] P.A. Grassi and G. Policastro, Super-Chern-Simons theory as superstring theory, hep-th/0412272 [INSPIRE].

[8] N. Berkovits and N. Nekrasov, Multiloop superstring amplitudes from non-minimal pure spinor formalism, JHEP 12 (2006) 029 [hep-th/0609012] [INSPIRE].

[9] C.A. Cremonini and P.A. Grassi, Pictures from Super Chern-Simons Theory, JHEP 03 (2020) 043 [arXiv: 1907.07152] [INSPIRE].

[10] L.N.S. Martins, Type IIB superstring vertex operator from the -8 picture, arXiv: 1912.06498 [INSPIRE].

[11] D. Friedan, E.J. Martinec and S.H. Shenker, Conformal Invariance, Supersymmetry and String Theory, Nucl. Phys. B 271 (1986) 93 [inSPIRE].

[12] A. Belopolsky, New geometrical approach to superstrings, hep-th/9703183 [INSPIRE].

[13] A. Belopolsky, Picture changing operators in supergeometry and superstring theory, hep-th/9706033 [INSPIRE].

[14] M.V. Movshev, The odd twistor transform in eleven-dimensional supergravity, arXiv: 1206.0057 [INSPIRE].

[15] M.B. Green, J.H. Schwarz and L. Brink, Superfield Theory of Type II Superstrings, Nucl. Phys. B 219 (1983) 437 [INSPIRE].

[16] P. Deligne et al. eds., Quantum fields and strings: A course for mathematicians. Vol. 1, 2, AMS Press, Providence U.S.A. (1999). 